\title{
LOW-COMPLEXITY BLIND MAXIMUM-LIKELIHOOD DETECTION FOR SIMO SYSTEMS WITH GENERAL CONSTELLATIONS
}

\author{
Weiyu Xu, Mihailo Stojnic and Babak Hassibi \\ Electrical Engineering Department, California Institute of Technology
}

\begin{abstract}
The demand for high data rate reliable communications poses great challenges to the next generation wireless systems in highly dynamic mobile environments. In this paper, we investigate the joint maximum-likelihood (ML) channel estimation and signal detection problem for single-input multiple-output (SIMO) wireless systems with general modulation constellations and propose an efficient sequential decoder for finding the exact joint ML solution. Unlike other known methods, the new decoder can even efficiently find the joint ML solution under high spectral efficiency non-constantmodulus modulation constellations. In particular, the new algorithm does not need such preprocessing steps as Cholesky or QR decomposition in the traditional sphere decoders for joint ML channel estimation and data detection. The elimination of such preprocessing not only reduces the number of floating point computations, but also will potentially lead to smaller size and power consumption in VLSI implementations while providing better numerical stability.
\end{abstract}

Index Terms - blind ML detection, decoding complexity, SIMO, maximum-likelihood,sphere decoder

\section{INTRODUCTION}

The goal of achieving high-speed reliable data transmission over highly dynamic wireless medium has generated a lot of research activities in the communications and signal processing community. One of the largest challenges arising in wireless communications is how to deal with the wireless fading phenomenon, where the wireless channels may vary over time. In wireless communications, one often assumes knowledge of the channel coefficients at the receiver side by channel estimation from training sequences, but sending training symbols will sacrifice a fraction of the transmission rate. In wireless mobile systems, the channels may even change so rapidly that training and channel tracking will become infeasible. One possible solution is to differentially encode the transmitted data and thus eliminate the need for channel knowledge. Another solution is to do blind or semi-blind detection over the time-varying wireless channels, which has been shown to enhance the system performance considerably and often perform better than differential modulations $[1,2,3,4,5,6]$.

We here consider the problem of joint ML channel estimation and data detection for SIMO systems, where the transmitter uses only one antenna but the receiver employs multiple antennas. This problem was shown to be an integer least squares problem for SIMO systems using constant modulus constellations in [2], where the sphere decoder was originally proposed for finding the joint ML solution. In [4], similar ideas of sphere decoders were used for blind detection of orthogonal space-time block codes (OSTBC). There the sphere decoders and semidefinite relaxation approach (SDR) are shown to be two comparable blind detection methods for OSTBC in terms of performance and complexity although SDR is only an approximation method. Again, only BPSK and QPSK modulations are admissible in sphere decoders and SDR methods in [4]. In [9], the authors showed that the joint optimal estimation and detection problem admits certain polynomial algorithm for constant energy constellations.

Despite all these developments, there are two limitations on the algorithms for blind ML detection of SIMO systems in the literature. Firstly, these algorithms are only for constant-modulus modulation of small alphabet sizes. In fact, the problem of blind ML detection for non-constant-modulus modulation constellation can not be transformed into a standard integer least squares problem as in [2] [4], which makes sphere decoders inapplicable to the blind ML detection. However, in order to achieve high spectral efficiency, high-order modulation constellations are of great interest, especially for SIMO wireless systems where receive diversity enables SNR high enough to support these high-order constellations. To the best of our knowledge, there are no working algorithms with numerical results reported in the literature for blind exact $M L$ detection in high-dimensional SIMO wireless systems employing non-constantmodulus constellations. (A conceptual blind exact ML decoder for non-constant-modulus constellations was discussed without numerical results in [10] by the first author of the current paper. Although that method avoids exhaustive search, it still does not work efficiently for such high-dimensional problems considered in this paper.) Secondly, for the sphere decoders in [3] and [4], such preprocessing steps as Cholesky (or QR) decomposition are needed, which add greatly to the whole detection complexity. In fact, for VLSI implementations, similar preprocessing requirements may prove to be a significant hindrance to the feasibility of sphere decoders in terms of area and power consumption[7] [8]. Also, Cholesky or QR decomposition is numerically undesirable for VLSI implementations since it requires a lot of divisions and square root operations[7][8].

In this paper, we try to resolve these two limitations by proposing a new optimal joint channel estimation and signal detection algorithm which works efficiently, in some cases more efficient than traditional sphere decoders ( and also approximate methods like SDR). The new algorithm works for general constellations and does not need either Cholesky or QR decomposition. Analysis shows that for any data length, if the SNR is high enough, the average complexity of the algorithm will approach a constant times the data length while achieving the optimal performance. Simulation results verify the efficiency and effectiveness of the new algorithm.

\section{THE JOINT CHANNEL ESTIMATION AND SIGNAL DETECTION PROBLEM}

Let us consider a SIMO system with $N$ receive antennas and let $T$ be the length of a data packet during which the channel remains static. 
Then the channel output is written as

$$
X=\mathbf{h s}^{*}+W,
$$

where $\mathbf{h} \in \mathcal{C}^{N \times 1}$ is the SIMO channel vector, $\mathbf{s}^{*} \in \mathcal{C}^{1 \times T}$ is the transmitted symbol sequence, and $W \in \mathcal{C}^{N \times T}$ is an additive noise matrix whose elements are assumed to be i.i.d. complex Gaussian random variables. And we also assume the entries of $\mathbf{s}^{*}$ are i.i.d. symbols from a certain constellation $\Omega$ (like QPSK or 16-QAM) of unit expected energy, i.e.,

$$
E\left(\left|\mathbf{s}_{k}\right|^{2}\right)=1, k=1,2, \ldots, T .
$$

We assume a deterministic unknown channel model for $\mathbf{h}$ where $\mathbf{h}$ is a deterministic unknown and no priori information about $\mathbf{h}$ is known [1][4]. This deterministic unknown channel model is preferred in some scenarios than assuming an i.i.d. Rayleigh distributed channel gains since sometimes wireless channels obey Rician fading, Nakagami or other fading statistics and there are possibly correlations between channel gains across the $N$ receive antennas. In fact, if we assume that $\mathbf{h}$ is a complex Gaussian distributed, the joint ML channel estimation and data detection problem can still be transformed to a special case where the techniques for this deterministic unknown channel model still apply.

Under these assumptions, the problem of joint ML channel estimation and data detection for SIMO channels is transformed into the following optimization problem

$$
\min _{\mathbf{h}, \mathbf{s}^{*} \in \Omega^{T}}\left\|X-\mathbf{h} \mathbf{s}^{*}\right\|^{2},
$$

where $\Omega^{T}$ denotes the the set of $T$-dimensional signal vector. In fact for any given transmitted symbol $s^{*}$, the channel vector $\mathbf{h}$ that minimizes (3) can be shown to be

$$
\hat{\mathbf{h}}=X \mathbf{s}\left(\mathbf{s}^{*} \mathbf{s}\right)^{-1}=X \mathbf{s} /\|\mathbf{s}\|^{2},
$$

Substituting (4) into (3), we get

$$
\| X(\underbrace{\left.I-\frac{1}{\|\mathbf{s}\|^{2}} \mathbf{s s}^{*}\right)}_{=P_{\mathbf{s}}} \|^{2}=\operatorname{tr}\left(X P_{\mathbf{s}} X^{*}\right)=\operatorname{tr}\left(X X^{*}\right)-\frac{1}{\|\mathbf{s}\|^{2}} \mathbf{s}^{*} X^{*} X \mathbf{s},
$$

If the modulation constellation is of constant modulus (such as QPSK), the minimization of (5) over $\mathbf{s}$ is equivalent to solving the following problem:

$$
\max _{\mathbf{s} \in \Omega^{T}} \mathbf{s}^{*} X^{*} X \mathbf{s},
$$

If $\rho$ is larger than the maximum eigenvalue of $X^{*} X,(6)$ is equivalent to the following minimization problem (for constant modulus constellation)

$$
\min _{\mathbf{s} \in \Omega^{T}} \mathbf{s}^{*}\left(\rho I-X^{*} X\right) \mathbf{s},
$$

The optimization problem in (7) is an integer least-squares problem and the sphere decoding algorithm can solve it much more efficiently than exhaustive search. Actually, the sphere decoder chooses a search radius $r$ and restricts the search to the hypersphere.

$$
\mathbf{s}^{*}\left(\rho I-X^{*} X\right) \mathbf{s} \leq r^{2} \text {. }
$$

The readers are referred to [2] for details of sphere decoding procedures. However, traditional sphere decoding algorithm needs to do the Cholesky factorization of the matrix $\left(\rho I-X^{*} X\right)$ first in order to find the closest lattice point. This will introduce computational complexity in the order of $O\left(T^{2} N\right)$, which is not desirable for this application. Also, as seen from (5), for non-constant-modulus constellations, the blind ML detection problem will be different from (6) and thus can not be changed to integer least squares problems, preventing the application of sphere decoders.

\section{SOLVING THE JOINT PROBLEM WITH THE NEW ALGORITHM}

We start by considering the original problem given in (3). For $X, \mathbf{s}^{*}$ and $W$, denote the parts corresponding to their first $i(1 \leq i \leq T)$ time indices as $X_{(i)}, s_{(i)}^{*}$ and $W_{(i)}$ while $X_{i}, s_{i}^{*}$ and $\bar{W}_{i}$ will be reserved for their part corresponding to exactly the $i$-th time index.

Let us consider a partial data sequence $s_{(i)}^{*}$ up to the time index $i$ and define $M_{s_{(i)}^{*}}$ as

$$
M_{s_{(i)}^{*}}=\operatorname{tr}\left(X_{(i)} X_{(i)}^{*}\right)-\frac{1}{\left\|\mathbf{s}_{(i)}^{*}\right\|^{2}} \mathbf{s}_{(i)}^{*} X_{(i)}^{*} X_{(i)} \mathbf{s}_{(i)},
$$

Lemma 1 Let $R$ be the optimal value for the optimization problem in (3). If $M_{s_{(i)}^{*}}>R$, then $\mathbf{s}_{(i)}^{*}$ can not be the first $i$ symbols of the ML solution $\hat{\mathbf{s}}^{*}$ to (3).

Proof: Suppose $\mathbf{s}_{(i)}^{*}=\hat{\mathbf{s}}_{(i)}^{*}$ and we denote the optimal channel gains corresponding to $\hat{\mathbf{s}}^{*}$ as $\hat{\mathbf{h}}$. Then

$$
\begin{aligned}
R & =\left\|X_{(i)}-\hat{\mathbf{h}} \hat{\mathbf{s}}_{(i)}^{*}\right\|^{2}+\sum_{j=i+1}^{T}\left\|X_{j}-\hat{\mathbf{h}} \hat{\mathbf{s}}_{j}^{*}\right\|^{2} \\
& \geq \min _{\mathbf{h}}\left\|X_{(i)}-\mathbf{h} \hat{\mathbf{s}}_{(i)}^{*}\right\|^{2}+\sum_{j=i+1}^{T}\left\|X_{j}-\hat{\mathbf{h}} \hat{\mathbf{s}}_{j}^{*}\right\|^{2} \\
& \geq \min _{\mathbf{h}}\left\|X_{(i)}-\mathbf{h} \hat{\mathbf{s}}_{(i)}^{*}\right\|^{2}=M_{\hat{\mathbf{s}}_{(i)}^{*}}=M_{\mathbf{s}_{(i)}^{*}},
\end{aligned}
$$

where the last two equalities are due to (5). But $M_{\mathbf{s}_{(i)}^{*}}$ is larger than $R$, which forms a contradiction.

From Lemma 1, if the value $R$ of the optimization problem (3) can be estimated, we can restrict the search of the blind ML solution $\hat{\mathbf{s}}^{*}$ to the offsprings of those partial sequences $\mathbf{s}_{(i)}^{*}$ which satisfy $M_{\mathbf{s}_{(i)}^{*}}<R$. This motivates our proposed depth-first search algorithm for finding the blind ML solution. In the following algorithm description, we denote the $k$-th constellation point in the modulation constellation $\Omega$ as $\Omega(k)$.

\section{Exact Blind ML Detection Algorithm}

Input: the received channel output $X$, the search radius $r$, the modulation constellation $\Omega$ and a $1 \times T$ index vector $I$.

1. Set $i=1, r_{i}=r, I(i)=1$ and set $s_{i}^{*}=\Omega(I(i))$.

2. (Computing the bounds) Compute the metric $M_{\mathbf{s}_{(i)}^{*}}$.If $M_{\mathbf{s}_{(i)}^{*}}>$ $r$, go to 3; else, go to 4;

3. (Backtracking) Find the largest $1 \leq j \leq i$ such that $I(j)<$ $|\Omega|$. If there exists such $j$, set $i=j$ and go to 5 ; else go to 6 .

4. If $i=T$, store current $s_{(T)}^{*}$, update $r=M_{\mathbf{s}_{(T)}^{*}}$ and go to 3 ; else set $i=i+1, I(i)=1$ and $s_{i}^{*}=\Omega(I(i))$, go to 2 .

5. Set $I(i)=I(i)+1$ and $s_{i}^{*}=\Omega(I(i))$. Go to 2 .

6. If any sequence $s_{(T)}^{*}$ is ever found in Step 4, output the latest stored full-length sequence as ML solution; otherwise, double $r$ and go to 1 .

\subsection{Choice of the initial radius $r$}

The structure of the new blind ML algorithm easily suggests a probabilistic choice of the search radius. We know that $\|W\|^{2}$ is chisquare distributed with $2 N T$ degrees of freedom, it is natural to choose the radius $r$ such that $P\left(\|W\|^{2}>r^{2}\right) \leq 1-\epsilon$. Since the solution $R$ to the optimization problem in (3) is sure to be smaller than $\|W\|^{2}$, we will guarantee finding the blind ML data sequence with probability at least $1-\epsilon$ under this initial radius. 


\subsection{Constant update complexity per tree node w.r.t. $T$}

As we can see, the new joint ML decoder almost only involves additions and multiplications in the decoding process. The main source of complexity comes from Step 2 where the metric $M_{\mathbf{s}_{(i)}^{*}}$ is computed, which at first sight needs computational efforts $O\left(T^{2}\right)$. Instead, we propose to efficiently update $M_{\mathbf{s}_{(i)}^{*}}$ at each tree node with constant computational costs (as a linear function of $N$ ) by propagating the value $A_{i}$, which is defined as $A_{i}=\sum_{j=1}^{i} X_{j} \mathbf{s}_{j}$. Then we can update $A_{i+1}$ sequentially as $A_{i+1}=A_{i}+X_{i+1} \mathbf{s}_{i+1}$ and compute $M_{\mathbf{s}_{(i+1)}^{*}}=\operatorname{tr}\left(X_{(i+1)} X_{(i+1)}^{*}\right)-\left\|A_{i+1}\right\|^{2}$. Note that $\operatorname{tr}\left(X_{(i)} X_{(i)}^{*}\right)$, namely the energy of $X_{(i)}$ can be sequentially precomputed at the beginning of the algorithm and used in the whole search. So the computational cost of updating each tree node's metric does not depend on the search dimension $T$.

\section{COMPUTATIONAL COMPLEXITY}

In this section we provide an upper bound on the expected value of the number of arithmetic operations required by our algorithm. Given that the number of the arithmetic operations performed per each node of the search tree is roughly a constant multiple of $N$, it suffices to find the expected number of the nodes inside the search sphere, which further reduces to finding the expected number of the nodes per each level remaining inside the search sphere. To simplify analysis, we assume i.i.d. complex Gaussian channel gains as $\sqrt{\frac{\rho}{c}} \overrightarrow{\mathbf{h}}$, where $\rho$ is the SNR, $\overline{\mathbf{h}}$ is a vector with i.i.d. complex Gaussian random variables of unit variance and $c$ is the average energy of each component of the transmitted vector $\hat{\mathbf{s}}$. Let the expected number of the nodes at $i$-th level of the search tree be $C_{i}$. Recall that every node in the tree has a sequence of fixed symbols which leads to that node. Let $\overline{\mathbf{s}}_{(i)} \neq \hat{\mathbf{s}}_{(i)}$ be the sequence of symbols leading to the node of $i$-th level which has the highest probability of being in the search sphere. Let $P_{i}$ be the probability that this node is in the sphere. Then it is easy to see that

$$
C_{i} \leq 1+\left(q^{i}-1\right) P_{i}
$$

where $q$ denotes how many symbols we have in our constellation. From (11) it clearly follows that establishing an upper bound on $P_{i}$ is sufficient to establish an upper bound on $C_{i}$.

From the description of our algorithm it follows that

$$
P_{i}=\operatorname{Pr}\left(\left\|X_{(i)}\left(I-\frac{\overline{\mathbf{s}}_{(i)} \overline{\mathbf{s}}_{(i)}^{*}}{\left\|\overline{\mathbf{s}}_{(i)}\right\|^{2}}\right)\right\|_{F}^{2} \leq r^{2}\right)
$$

where $X_{(i)}$ denotes the first $i$ columns of the matrix $X$ and $r^{2}$ is the squared value of the search radius. We can further write

$$
P_{i}=\operatorname{Pr}\left(\operatorname{Tr}\left(X_{(i)}\left(I-\frac{\overline{\mathbf{s}}_{(i)} \overline{\mathbf{s}}_{(i)}^{*}}{\left\|\overline{\mathbf{s}}_{(i)}\right\|^{2}}\right) X_{(i)}^{*}\right) \leq r^{2}\right) .
$$

Recall that

$$
X_{(i)}=\sqrt{\frac{\rho}{c}} \overline{\mathbf{h}}_{(i)}^{*}+W_{(i)}
$$

where $W_{(i)}$ denotes first $i$ columns of the matrix $W$. Combining (12) and (13) we have

$$
P_{i}=\operatorname{Pr}\left(\operatorname{Tr}\left(\left[\begin{array}{c}
\overline{\mathbf{h}}^{*} \\
W_{(i)}^{*}
\end{array}\right]^{*} Q_{(i)}\left[\begin{array}{c}
\overline{\mathbf{h}}^{*} \\
W_{(i)}^{*}
\end{array}\right]\right) \leq r^{2}\right)
$$

where

$$
Q_{(i)}=\left[\begin{array}{c}
\sqrt{\frac{\rho}{c}} \hat{\mathbf{s}}_{(i)}^{*} \\
I
\end{array}\right]\left(I-\frac{\overline{\mathbf{s}}_{(i)} \overline{\mathbf{s}}_{(i)}^{*}}{\left\|\overline{\mathbf{s}}_{(i)}\right\|^{2}}\right)\left[\sqrt{\frac{\rho}{c}} \hat{\mathbf{s}}_{(i)} \quad I\right] .
$$

Using the Chernoff bound the right hand side of (14) can be bounded in the following way

$$
\begin{aligned}
P_{i} & \leq \min _{\mu \geq 0} e^{\mu r^{2}} E e^{-\mu \operatorname{Tr}\left(\left[\begin{array}{c}
\overline{\mathbf{h}}^{*} \\
W_{(i)}^{*}
\end{array}\right]^{*} Q_{(i)}\left[\begin{array}{c}
\overline{\mathbf{h}}^{*} \\
W_{(i)}^{*}
\end{array}\right]\right)} \\
& =\min _{\mu \geq 0} \int \frac{e^{-\operatorname{Tr}\left(\left[\begin{array}{c}
\overline{\mathbf{h}}^{*} \\
W_{(i)}^{*}
\end{array}\right]^{*}\left(I+\mu Q_{(i)}\left[\begin{array}{c}
\overline{\mathbf{h}}^{*} \\
W_{(i)}^{*}
\end{array}\right]\right)\right.} d \overline{\mathbf{h}}^{*} d W_{(i)}^{*}}{e^{-\mu r^{2} \pi^{N(i+1)}}} \\
& =\min _{\mu \geq 0} \frac{e^{\mu r^{2}}}{\operatorname{det}\left(I+\mu Q_{(i)}\right)^{N}} .
\end{aligned}
$$

It is straightforward to compute the determinant in the denominator

$$
\begin{aligned}
& \operatorname{det}\left(I+\mu Q_{(i)}\right)=\operatorname{det}\left(I+\mu\left(I+\frac{\rho}{c} \hat{\mathbf{s}}_{(i)} \hat{\mathbf{s}}_{(i)}^{*}\right)\left(I-\frac{\overline{\mathbf{s}}_{(i)} \overline{\mathbf{s}}_{(i)}^{*}}{\| \overline{\mathbf{s}}_{(i)}||^{2}}\right)\right) \\
& =(1+\mu)^{i-2}\left(1+\mu\left(1+\rho\left\|\hat{\mathbf{s}}_{(i)}\right\|^{2}\left(1-\frac{\left|\hat{\mathbf{s}}_{(i)}^{*} \overline{\mathbf{s}}_{(i)}\right|^{2}}{\left\|\hat{\mathbf{s}}_{(i)}||^{2}\right\| \overline{\mathbf{s}}_{(i)} \|^{2}}\right)\right)\right) .
\end{aligned}
$$

Combining (11), (15), and (16) we finally obtain an upper bound on the expected complexity $C_{i}$

$$
1+\min _{\mu \geq 0} \frac{\left(q^{i}-1\right) e^{\mu r^{2}}(1+\mu)^{2-i}}{\left(1+\mu\left(1+\rho\left\|\hat{\mathbf{s}}_{(i)}\right\|^{2}\left(1-\frac{\left|\hat{\mathbf{s}}_{(i)}^{*} \overline{\mathbf{s}}_{(i)}\right|^{2}}{\left\|\hat{\mathbf{s}}_{(i)} \mid\right\|^{2}\left\|\overline{\mathbf{s}}_{(i)}\right\|^{2}}\right)\right)\right)^{N}} .
$$

Setting $\mu=\frac{N i}{r^{2}}$ and assuming $\overline{\mathbf{s}}(i) \neq \hat{\mathbf{s}}(i)$

$$
\lim _{\rho \rightarrow \infty} C_{i} \leq 1 \text {. }
$$

This effectively means that for high enough SNR $\rho$ only nodes corresponding to the sequence $\hat{\mathbf{s}}$ will be in the search sphere. Since we perform $O(N)$ arithmetic operations per each node of the search tree the overall number of arithmetic operations will be $O(N T)$ which is even significantly smaller than $O\left(N T^{2}\right)$ required by the Cholesky factorization part of the sphere decoder algorithm.

\section{SIMULATION RESULTS}

In this section, we give simulation results for the performance and complexity of the new blind ML decoder. First, we investigates its performance and complexity in a SIMO system employing BPSK modulation where we assume $N=2$ and $T=21$. In the simulations, the channel coefficients are generated as i.i.d complex Gaussian random variables. To resolve the phase ambiguity, we embed one known symbol in the data block. In Figure 1 shows that the blind exact ML decoding performance obtained using the new blind ML decoder. In Figure 2, we show the average number of floatingpoint operations (FLOPS) for each detection block in the new algorithm. In fact, when $S N R$ is larger than $12 \mathrm{~dB}$, the new blind ML decoder even requires less computations than the preprocessing part of traditional sphere decoders[2]. In Figure 3, we give the BER performance of our algorithm for a SIMO system employing 16-QAM constellation where $N=6$ and $T=11$. It can be seen that the new algorithm works very close to the known channel case, while having huge performance advantage over iterative joint channel estimation and data detection. In the iterative scheme, the least-squares(LS) channel estimation was first initialized from the known embedded symbol based on (4) and then the so-obtained channel estimation 


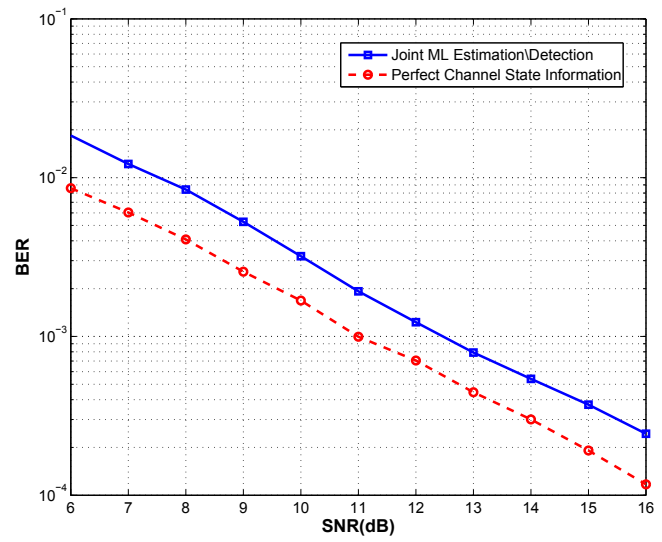

Fig. 1. BER Performance of SIMO BPSK System with $N=2$ and $T=21$

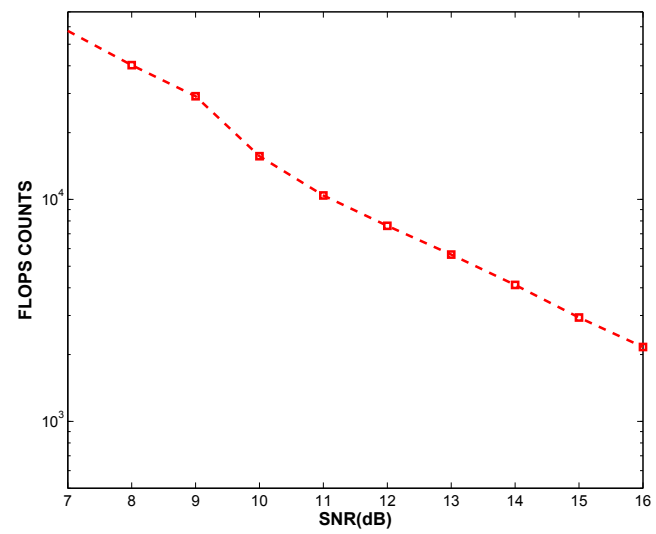

Fig. 2. Flop Counts for the New Blind ML Algorithm for BPSK Modulation with $N=2$ and $T=21$

was used for coherent data detection. After that, the channel is reestimated by further using the detection decisions. The estimationdetection procedure was iterated for a preimposed 20 times in the simulations. Notice for each block, we need to test $2^{4 \times 10} \approx 10^{12}$ hypothesis assumptions in exhaustive search for solving the problem (3), which is almost infeasible for getting this result.

\section{SUMMARY AND DISCUSSION}

In this paper, we propose a low-complexity sequential decoder which achieves the exact joint ML channel estimation and data detection for SIMO wireless systems with high-order non-constant-modulus modulation, where no other efficient exact methods (such as sphere decoders) apply. It is shown analytically that as the SNR grows, the average complexity of the new blind ML decoding algorithm will approach a constant times the data length $T$. The simple structure of the new decoder without Cholesky factorization will potentially lead to smaller size and power consumption in its VLSI implementation while providing better numerical stability.

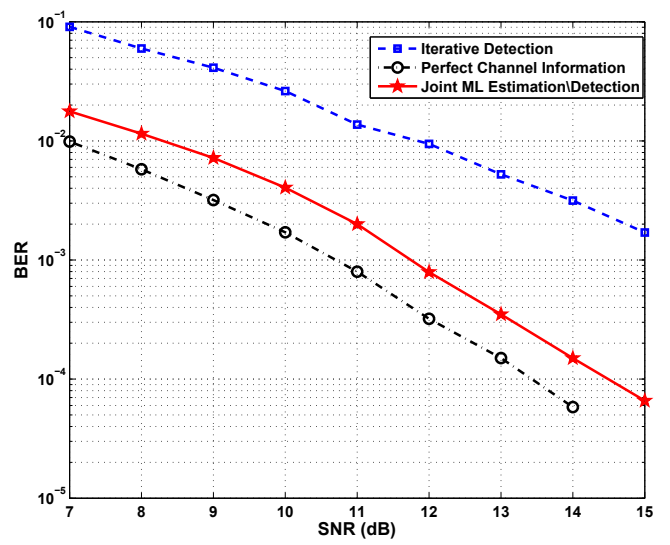

Fig. 3. Blind ML Decoding Performance for 16-QAM

\section{REFERENCES}

[1] P. Stoica and G. Ganesan, "Space-time block codes: Trained, blind, and semi-blind detection," Digital Signal Process., vol. 13, pp. 93-105, 2003.

[2] H. Vikalo, B. Hassibi and P. Stoica, " Efficient joint maximumlikelihood channel estimation and signal detection," IEEE Transactions on Wireless Communications, vol 5, no 7, pp. 1838-1845, Jul 2006.

[3] P.Stoica, H.Vikalo and B.Hassibi,"Joint maximum-likelihood channel estimation and signal detection for SIMO channels," in Proceedings of 2003 International Conference on Acoustics,speech and signal processing, vol.4,2003,pp. 13-16.

[4] W.-K. Ma, B.-N. Vo, T.N. Davidson, and P.C. Ching, "Blind ML detection of orthogonal space-time block codes: Highperformance, efficient implementations," IEEE Transactions on Signal Processing, vol. 54, no. 2, pp. 738-751, Feb. 2006.

[5] A. L.Swindlehurst and G. Leus, "Blind and semi-blind equalization for generalized space-time block codes," IEEE Trans. Signal Process., vol. 50, no. 10, pp. 2589-2498, 2002.

[6] S. Shahbazpanahi, A. B. Gershman, and J. H. Manton, "Closedform blind MIMO channel estimation for orthogonal space-time block codes," IEEE Trans. Signal Process., vol. 53, no. 12, pp. 4506-4517, Dec. 2005.

[7] G. Knagge, L. Davis, G. Woodward, S. R. Weller, "VLSI Preprocessing Techniques for MUD and MIMO Sphere Detection", Proceedings 6th Australian Communications Theory Workshop (AusCTW2005), pp. 204-210, Feb 2005.

[8] D. Garrett, L. Davis, S. ten Brink, B. Hochwald, and G. Knagge, "Silicon complexity for maximum likelihood MIMO detection using spherical decoding," IEEE J. Solid-State Circuits, vol. 39, no. 9, pp. 1544-1552, Sep. 2004.

[9] I.Motedayen, Arvind Krishnamoorthy and A.Anastaspopoulos, "Optimal Joint Detection/Estimation in Fading Channels with Polynomial Complexity," IEEE Transactions on Information Theory,vol. 53, no. 1,pp. 209-223 January 2007;

[10] Weiyu Xu, Y. Wang, Z. Zhou and J. Wang, "Joint ML channel estimation and data detection for STBC via novel sphere decoding algorithms", Proceedings of Vehicular Technology Conference, vol.1, pp. 434-437, Fall, 2005. 